
\title{
Allogeneic stem cell transplantation in CML patients with history of
accelerated phase or blast crisis: comparison of haploidentical and matched related stem cell transplantation
}

BadaevR., Zammoeva D., Babenetskaya D., I I ina N., Siordia N., Lazorko N., Ovsyannikova E. Alexe eva Y., Lomaia E., Zaritskey A., Motorin D.

\section{Objective}

Our primary goal was to evaluate outcome after haploidentical and matched related stem cell transplantation in CML patients with history of accelerated phase and blast crisis, performing retrospective analysis of patients who underwent alloSCT in our unit between 2010-2018

\section{Patients \& Methods}

$18 \mathrm{CML}$ patients with history of AP/BC were transplanted - 10 haploidentical and 8 matched related stem cell transplantations. Males/females were 6/4 In group of haploSCT and 5/3 in group of matched related SCT. Median age was $46(28-58)$ years and 35 (23-59) years respectively. Median time from initial diagnosis to AP/BC transformation was 48,5 (8-189) months in haploidentical and $7(0-123)$ months in matched related groups. Time from last $\mathrm{AP} / \mathrm{BC}$ to transplantation was 4,6 (0,6-9,9) months and 3,9 (0,7$94,3)$ months respectively.

On time of transplantation 7 patients were in $\mathrm{CP}, 1$ in $\mathrm{AP}$ and 2 in $\mathrm{BC}$ in haplo and $5 \mathrm{CP} \& 3 \mathrm{BC}$ in matched related SCT

Conditioning regimen for haploidentical SCT was RIC FluCyMel, GVHD prophylaxis with PTCY+cyclosporin+MMF. In matched related SCT conditioning regimen was MAC Bu16Cy120, GVHD prophylaxis: MTX+cyclosporin. In case of advanced stages conditioning regimen was with previous cytoreduction.

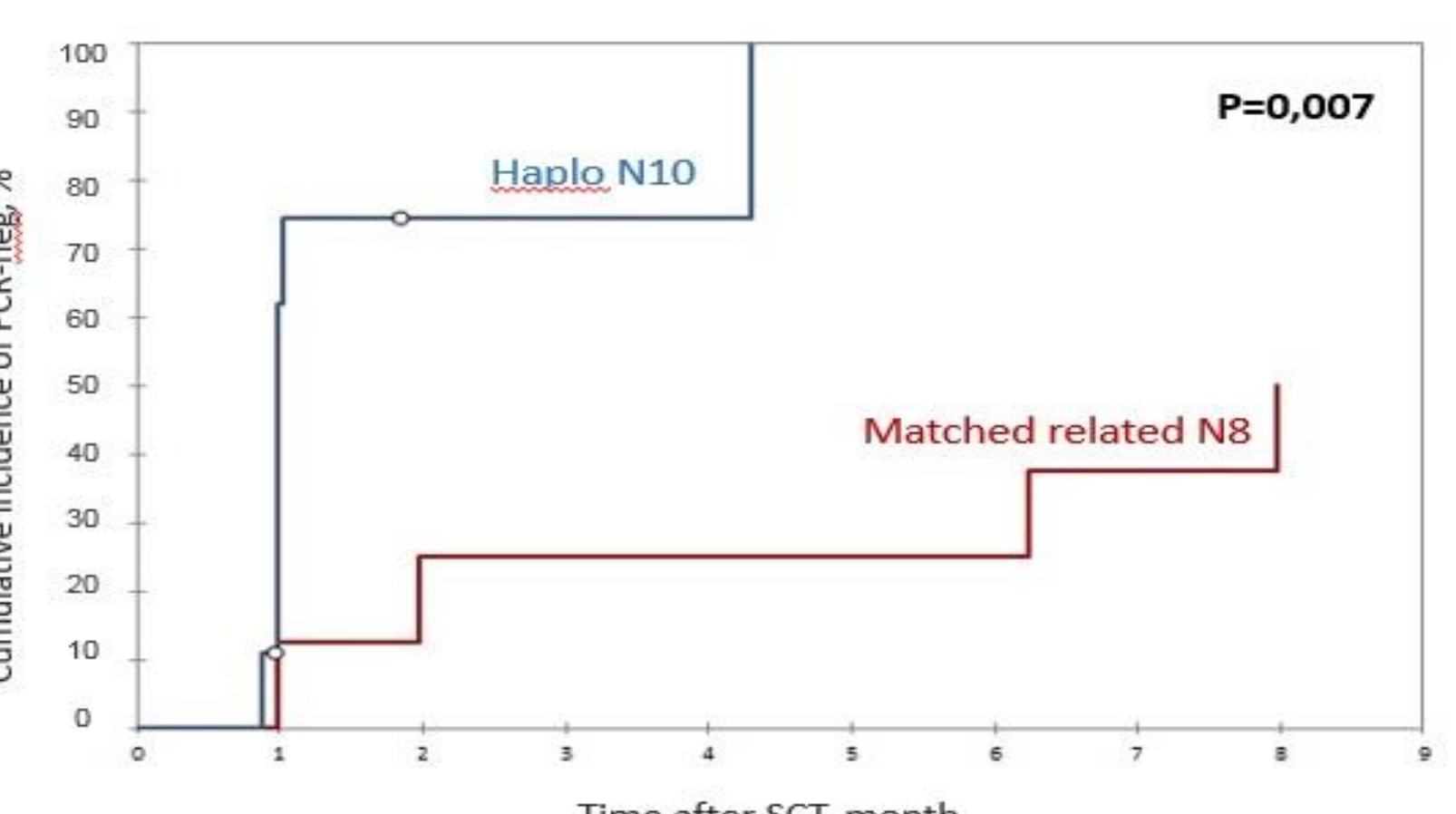

Time after SCT, month

Figure 1 - cumulative incidence of PCR-negativity

\section{Results}

Median follow up after haploidentical and matched related transplantation was 10,3 (0,3-42,4) months and 13,9 (1,3-61,4) months respectively. Transplant-related deaths occurred among $3 / 10$ patients in haploidentical SCT and 0/8 patients in matched related SCT groups, with no statistical difference $(p>0,05)$. In group of haploidentical stem cell transplantation $3 / 10$ patients died before engraftment: 1 patient with primary graft failure and 2 patients due to severe infections.

We evaluated response to treatment using cumulative incidence of achievement of PCR-negativity after SCT. Cumulative incidence of achieving PCR-negative status was higher after haploidentical SCT compared with matched related SCT group with statistically significant difference $(p=0,007)$. Among evaluable patients rate of PCR-negativity by D+30 was $5 / 7(70 \%)$ in haploidentical SCT and $1 / 8(12,5 \%)$ in matched related SCT groups, and by D+60 - 6/7 (85\%) and 3/8 (37,5\%) respectively, with strong tendency to be higher at haploidentical group by $\mathrm{D}+30(\mathrm{p}=0,073)$. PCR-negative status was achieved in $7 / 7(100 \%)$ after haploidentical stem cell transplantation and 6/8 $(75 \%)$ after matched related stem cell transplantation with median 1 (1-4,2) months and 2 (1-7) months respectively. Acute GVHD was observed in $7 / 7(100 \%)$ cases after haploidentical SCT and 4/8 (50\%) after matched related SCT, with gr III-IV in $3 / 7(42,8 \%)$ and $1 / 8$ (12,5\%) respectively. Extensive chronic GVHD was observed in 1/7 (14,3\%) after haploidentical SCT and 2/8 (25\%) after matched related SCT. No statistical difference in rates of severe acute and chronic GVHD was observed $(p>0,05)$. Rate of blastic phase relapse after SCT was 1/7 (14,3\%) after haploSCT and 4/8 (50\%) after matched related SCT, with no statistical difference $(p>0,05)$. All patients in relapse tapered immunosuppression and received TKI and DLI. Another 2 patients received TKI due to prolonged BCR-ABL persistence. Among patients with BC after SCT 4 died and 1 is alive with 2 nd transplant ongoing. Overall survival after haploidentical and matched related SCT is $50 \%$ and $62,5 \%$ respectively with no statistical difference $(p>0,05)$.

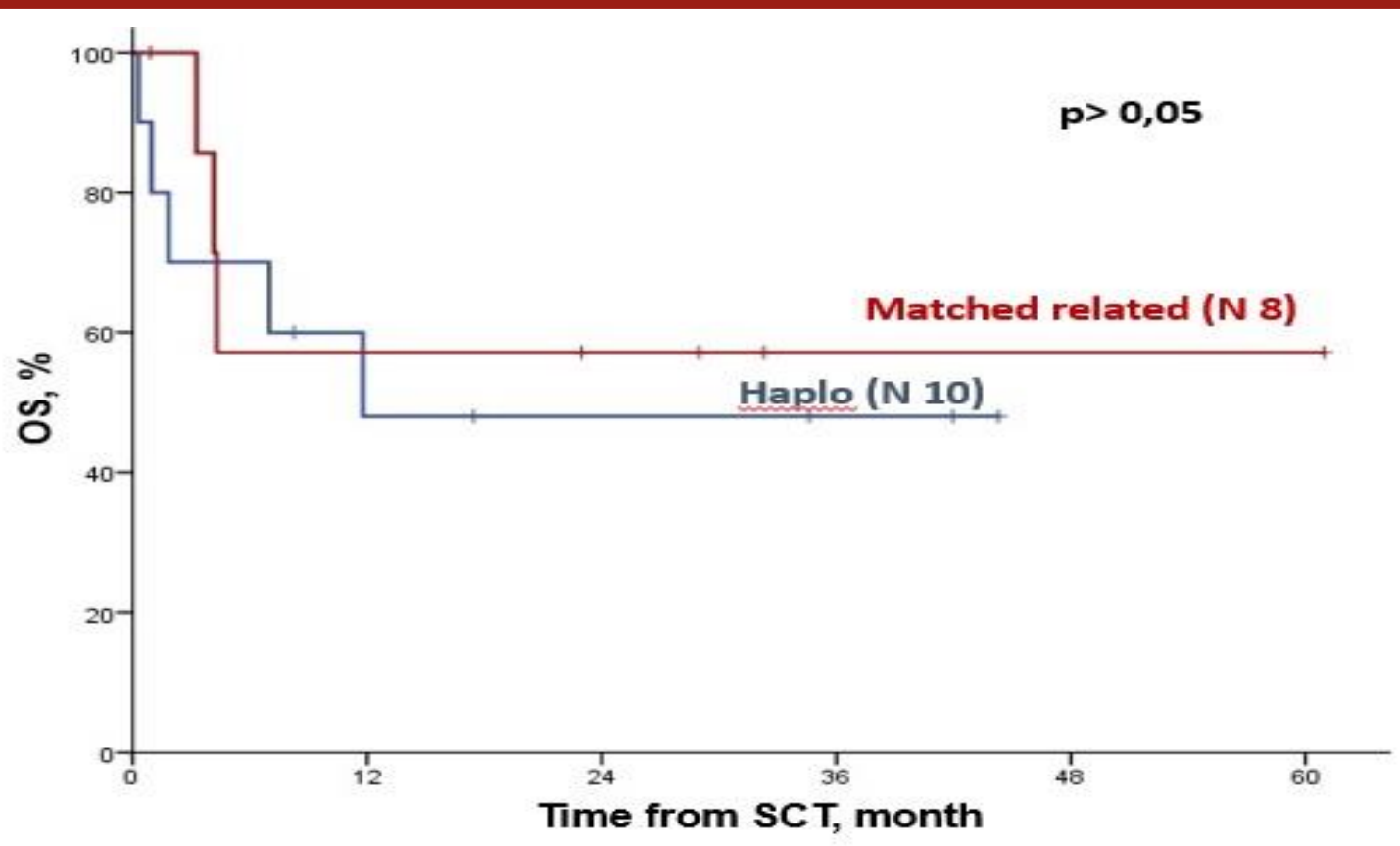

Figure .2 - Overall survival after haploidentical and matched related stem cell transplantation

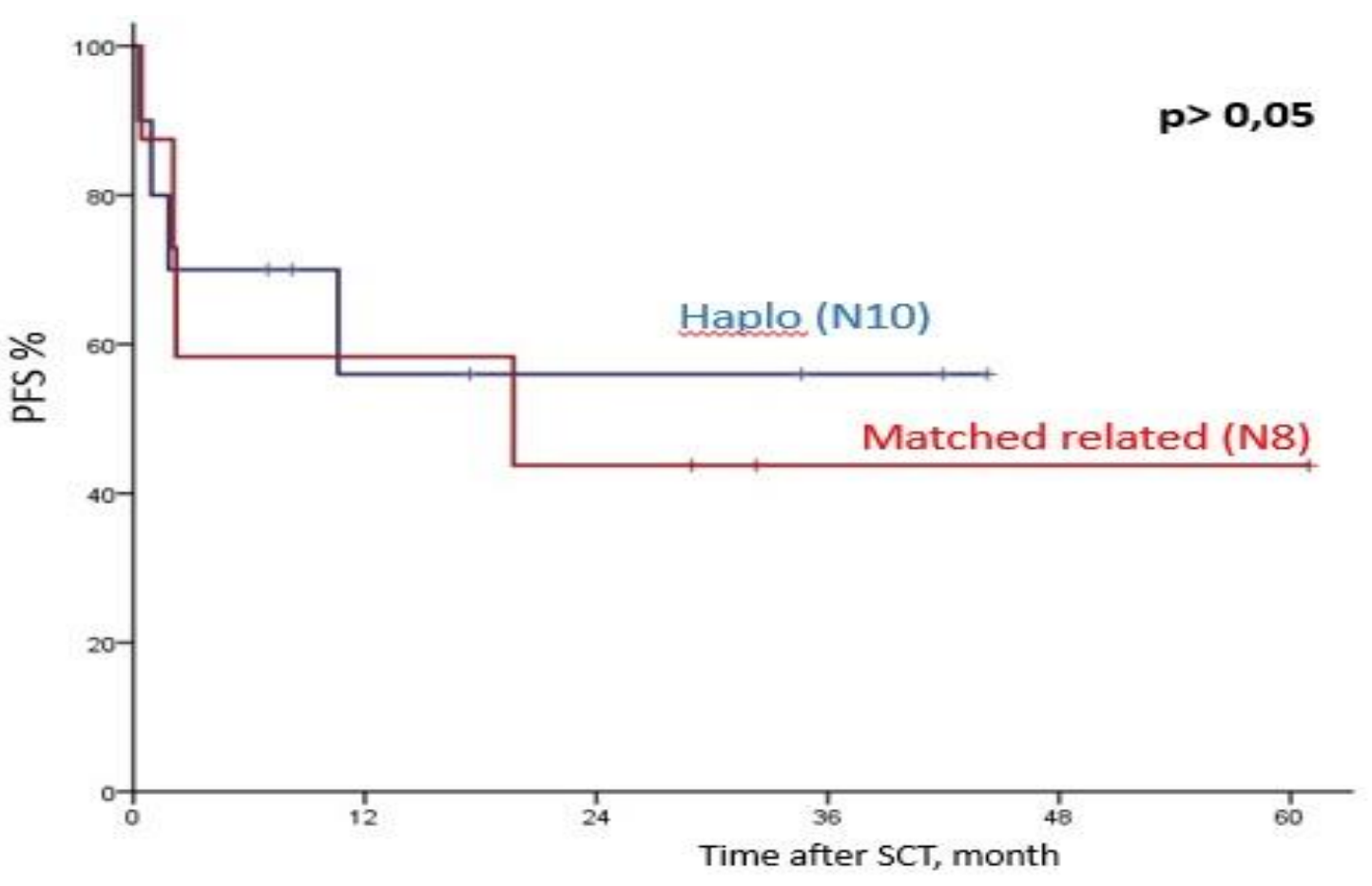

Figure .3 - Relapse-free survival after haploidentical and matched related stem cell transplantation

\section{Summary}

No difference in OS, RFS, acute and chronic GVHD rate was observed among matched related and haploidentical SCT recipients. We observed significantly higher rate of PCR-negativity achievement in group of haploSCT. Also we found lower rate of blastic phase relapses after haploidentical SCT compared with matched related SCT, but this difference is not statistically significant. We suggest that these results are related to more rapid and potent GVL effect in haploidentical SCT setting. Rate of transplant-related deaths was not significantly different. These data support that haploidentical SCT have comparable results with matched related SCT and is valuable alternative in patients with $\mathrm{CML} \mathrm{AP} / \mathrm{BC}$ with no matched related sibling. 